\title{
Tissue transglutaminase (TG2) protects cardiomyocytes against ischemia/reperfusion injury by regulating ATP synthesis
}

\author{
Cell Death and Differentiation (2006) 13, 1827-1829. doi:10.1038/sj.cdd.4401889; published online 10 March 2006
}

Dear Editor,

Apoptosis deregulation, since its discovery, has been shown to play an important role in the onset of numerous diseases including myocardial ischemia and reperfusion. ${ }^{1}$ Ischemic injury is thought to initiate apoptosis, but for substantial cell death to occur, reperfusion is necessary. ${ }^{1}$ Among many others the initial high-energy phosphate content of the heart were reported to affect the onset of cardiac cell apoptosis following ischemia/reperfusion. ${ }^{2}$

Transglutaminases are a family of thiol- and $\mathrm{Ca}^{2+}$. dependent acyl transferases that catalyze the formation of a covalent bond between the $\gamma$-carboxamide groups of peptidebound glutamine residues and various primary amines, including the $\varepsilon$-amino group of lysine in certain proteins. ${ }^{3}$ TG2, one of the eight distinct, enzymatically-active transglutaminases, is ubiquitously expressed in mammalian tissues. ${ }^{3}$ The enzyme has been implicated in a variety of cellular processes including cell death. ${ }^{3}$ In addition to its cross-linking activity, TG2 also acts as GTP-binding protein mediating intracellular signaling via the alpha-1b-adrenergic receptor $(\mathrm{AR}) .{ }^{3}$ Recently a protein disulphide isomerase and a kinase activity of TG2 have also been described. ${ }^{4,5}$

To assess the biological role, TG2 knockout mice have been generated. These mice show impaired glucose-stimulated insulin secretion and intolerance after glucose loading. ${ }^{6}$ The resulting phenotype resembles that of maturity onset diabetes of young (MODY) suggesting that TG2 might play a physiological role in glucose tolerance. ${ }^{6}$ Defects in glucoseinduced insulin release have been widely related to defective respiratory chain activation and loss of ATP production. ${ }^{7}$ This, however, has never been investigated in the context of TG2 deficiency, despite of the fact that TG2 colocalizes with mitochondria, and its overexpression leads to mitochondrial hyper-polarization. ${ }^{8}$ In addition, by studying these mice it was also found that TG2 acting as G protein in the AR signaling pathway protects hepatocytes against Fas-mediated death. ${ }^{9}$ As AR is also expressed by cardiac cells and Fas signaling was shown to participate in heart failure,${ }^{1}$ we decided to study the impact of TG2 on cardiac ischemia/reperfusion injury.

To this aim wild-type (WT) and TG $2^{-l-}$ hearts were isolated and aerobically perfused for $30-$ min of preischemic period. At the end of this period control measurements of electrocardiogram, heart rate $(\mathrm{HR})$, coronary flow $(\mathrm{CF})$, aortic flow $(\mathrm{AF})$, and aortic pressure (AOP) were recorded. Global ischemia was then induced for $40 \mathrm{~min}$ followed by $2 \mathrm{~h}$ of reperfusion. Cardiac functions were monitored after 30, 60 and $120 \mathrm{~min}$ following reperfusion and the size of the infarct was determined at the end of the reperfusion period. As shown in Figure 1a, the infarct size was significantly increased $(P<0.05)$ in the $\mathrm{TG}^{-1-}$ ischemic/reperfused hearts $(47.2 \pm 3.3 \%)$ as compared to the drug-free ischemic/reperfused WT group $(37.2 \pm 2.1 \%)$. (Figure 1a). Similarly to the infarct size, the incidence of reperfusion-induced ventricular fibrillation was also significantly increased $(P<0.05)$ in the $\mathrm{TG}^{-1-}$ ischemic/reperfused hearts (83\%) as compared to the WT ischemic/reperfused group (33\%) resulting in a significant worsening in the recovery of the postischemic cardiac function in $\mathrm{TG} 2^{-l-}$ mice. For instance, after $40 \mathrm{~min}$ of ischemia followed by $120 \mathrm{~min}$ of reperfusion, AF was only $0.8 \pm 0.1 \mathrm{ml} /$ min in $\mathrm{TG}^{-1-}$ hearts, while it was $2.6 \pm 0.4 \mathrm{ml} / \mathrm{min}$ in WT hearts $(P<0.05)$. It is important to note, however, that already the preischemic values of $\mathrm{HR}, \mathrm{CF}, \mathrm{AF}$ and AOP of the TG2 ${ }^{-1-}$ control group were significantly $(P<0.05)$ reduced when compared to those of the WT control values (data not shown).

Since TG2 participates in the AR signaling pathway in cardiac cells, we decided to test whether impaired AR signaling is responsible for the observed increased ischemic sensitivity of $\mathrm{TG}^{-/-}$hearts. To achieve this, hearts isolated from AR null and their WT counterpart mice were exposed to the treatments described above. However, no significant difference was found in the infarct size, the incidence of ventricular fibrillation, or the postischemic cardiac functional parameters between these strains (data not shown), indicating that the increased sensitivity of $\mathrm{TG}^{-1-}$ hearts to ischemia/ reperfusion injury is not related to an impaired $A R$ signaling.

Considering that the functional parameters of $\mathrm{TG}^{-/-}$ hearts were steady-state much worse than that of the WTs, and high-energy phosphate content was reported to affect the cardiac functional parameters before and after ischemia/ reperfusion, ${ }^{2}$ we decided to test the high-energy phosphate content of the WT and TG2 ${ }^{-1-}$ hearts before and after ischemia/reperfusion. As shown in Figure 1b, there was a significant reduction in the high-energy phosphate content of the $\mathrm{TG} 2^{-1-}$ mice already before ischemia which was further reduced following ischemia/reperfusion. These data imply that in $\mathrm{TG}^{-/-}$hearts the maintenance of the high-energyphosphate content is impaired. This defect could reflect a 
a

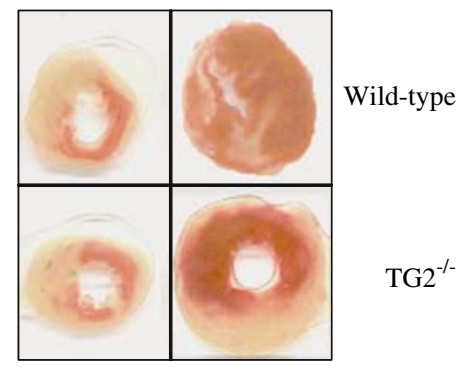

b

Effect of ischemia/reperfusion on high-energy phosphate content of TG2 ${ }^{-/}$and C57BL/6mice myocardium

\begin{tabular}{|c|c|c|c|c|}
\hline \multicolumn{5}{|c|}{ Before ischemia } \\
\hline \multirow[b]{2}{*}{$\begin{array}{l}\text { C57BL/6 } \\
\text { TG2 }^{-/-}\end{array}$} & ATP & $\begin{array}{l}\text { ADP } \\
(\mu \mathrm{mol} / \mathrm{g}\end{array}$ & $\begin{array}{c}\text { AMP } \\
\text { weight) }\end{array}$ & $\mathrm{CrP}$ \\
\hline & $\begin{array}{l}6.18 \pm 2.52 \\
3.37 \pm 1.32^{*}\end{array}$ & $\begin{array}{c}0.34 \pm 0.09 \\
0.17 \pm 0.05\end{array}$ & $\begin{array}{l}0.073 \pm 0.027 \\
0.039 \pm 0.011^{*}\end{array}$ & $\begin{array}{l}6.48 \pm 0.51 \\
4.55 \pm 1.07\end{array}$ \\
\hline \multicolumn{5}{|c|}{ After reperfusion } \\
\hline & ATP & $\begin{array}{l}\text { ADP } \\
(\mu \mathrm{mol} / \mathrm{g}\end{array}$ & $\begin{array}{r}\text { AMP } \\
\text { weight) }\end{array}$ & $\mathrm{CrP}$ \\
\hline $\begin{array}{l}\text { C57BL/6 } \\
\text { TG2 } 2^{-/-}\end{array}$ & $\begin{array}{l}3.81 \pm 2.41 \\
2.00 \pm 1.20\end{array}$ & $\begin{array}{l}0.36 \pm 0.12 \\
0.13 \pm 0.04\end{array}$ & $\begin{array}{c}0.024 \pm 0.011 \\
0.029 \pm 0.011\end{array}$ & $\begin{array}{l}5.20 \pm 1.43 \\
3.36 \pm 1.11^{*}\end{array}$ \\
\hline
\end{tabular}

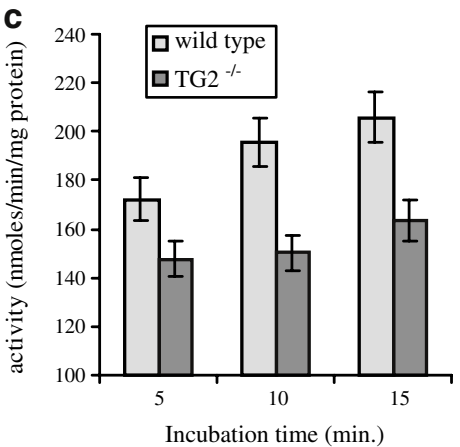

d

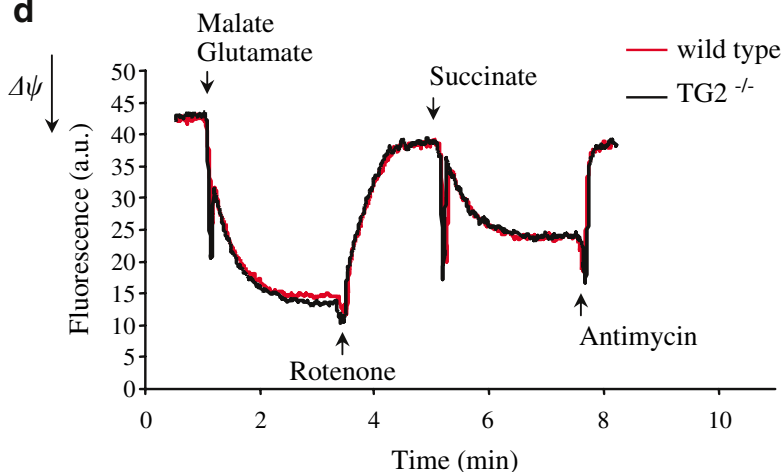

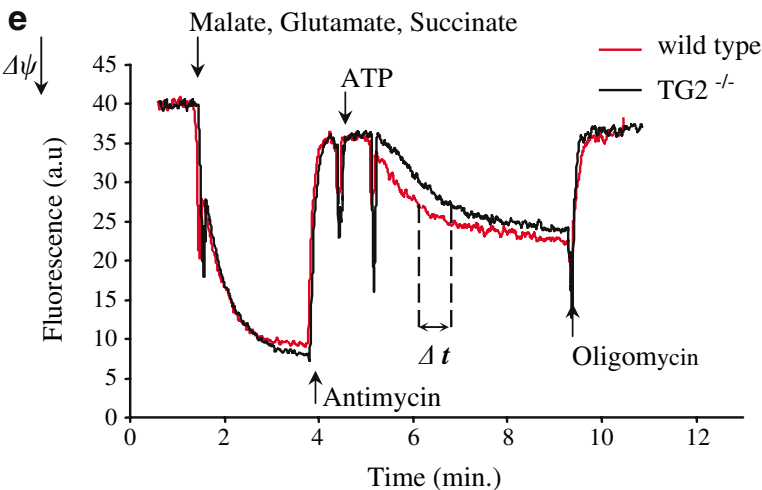

Figure 1 (a-b) Male mice (25-35 g), $\mathrm{TG}^{-1-}, \mathrm{AR}^{-1-}$ and their WT (C57Bl6) littermates were used for all studies. Hearts were rapidly excised and mounted to a Hewett 'working' perfusion apparatus. An epicardial ECG was recorded throughout the experimental period by two silver electrodes attached directly to the heart. The ECGs were analyzed to determine the presence or absence of reperfusion-induced VF. (a) Representative pictures of infarct size limitation in hearts of WT and TG2 ${ }^{-1-}$ mice after $40 \mathrm{~min}$ ischemia followed by $120 \mathrm{~min}$ reperfusion. Hearts were perfused at the end of each experiment with $15 \mathrm{ml}$ of $1 \%$ triphenyl tetrazolium solution in phosphate buffer via the side arm of the aortic cannula, and stored at $-70^{\circ} \mathrm{C}$ for later analysis. Frozen hearts were sliced transversely in a plane perpendicular to the apico-basal axis into 2-3 mm thick sections, weighted, blotted dry, placed in between microscope slides and scanned on a Hewlett-Packard Scanjet $5 p$ single pass flat bed scanner. The areas were measured by computerized planimetry software and these areas were multiplied by the weight of each slice, then the results summed up to obtain the weight of the risk zone (total weight of the left ventricle, $\mathrm{mg}$ ) and the infarct zone (mg). Infarct size was expressed as the ratio, in percent, of the infarct zone to the risk zone. The infarcted area is shown as white area in ischemic/reperfused mouse heart sections stained by $1 \%$ triphenyl tetrazolium solution. (b) Adenine nucleotide contents from WT and TG2 ${ }^{-l-}$ hearts after $40 \mathrm{~min}$ ischemia followed by $120 \mathrm{~min}$ reperfusion. For HPLC analysis ATP, ADP and AMP were separated on a reverse phase $\mathrm{C}_{18}$ column (LiChrosorb $5 \mathrm{RP} 18,1504.5 \mathrm{~mm} 1 / 4^{\prime \prime}$ Valco, Varian Co). The data were collected, and the results were expressed in $\mu$ mol/g wet weight. (c) Measure of ATP synthesis in TG2 ${ }^{-l-}$ versus WT mitochondria. To assess whether defect of ATP hydrolysis was paralleled by an anomaly in the reverse reaction, ATP synthesis from its precursor ADP, rate of ATP production was monitored by the indirect method of Beil. ${ }^{10}(\mathbf{d}-\mathrm{e})$ Defective heart mitochondrial respiratory chain function in TG2 ${ }^{-l-}$ mice. Mitochondrial membrane potential $\Delta \psi$ was measured in isolated mitochondria by monitoring $(8 \mu \mathrm{M})$ safranine 0 emission in a Perkin Elmer Luminescence Spectrometer at 495 and $586 \mathrm{~nm}$ excitation and emission wavelengths, respectively. (d) To measure the mitochondrial potential building (5 mM) malate and (5 mM) glutamate, substrates for complex I, were added to purified heart mitochondria; $\Delta \psi$ was built by complexes I-III-IV and Safranine 0 emission decreased. Following addition of $(1.3 \mu \mathrm{M})$ rotenone, inhibitor of complex I, $\Delta \psi$ readily returned to the basal level. Addition of $(5 \mathrm{mM})$ succinate, substrate for complex II, caused a new polarization due to complexes II-III-IV activity. Following addition of $(1.3 \mu \mathrm{M})$ antimycin, inhibitor of complex III, $\Delta \psi$ returned again to the basal level. Lacking of differences in Safranine 0 emission indicated no differences between the activity of complexes I-III-IV and II-III-IV of TG2 ${ }^{-1-}$ and control mice. (e) To analyze the ATPase activity (5 mM) malate, $(5 \mathrm{mM})$ glutamate and $(5 \mathrm{mM})$ succinate were added to purified mitochondria, and the membrane potential was readily built by complexes I-III-IV and II-III-IV activities to indicate that mitochondria were active. After addition of antimycin, inhibitor of complex III, $\Delta \psi$ rapidly decreased. Addition of (1 mM) ATP caused a novel potential building due to ATP-synthase proton pumping followed by ATP hydrolysis to ADP

failure in mitochondrial ATP production. To test this possibility, we decided to measure the membrane polarization $(\Delta \psi)$ on mitochondria isolated from hearts of control and $\mathrm{TG}^{-{ }^{-}-}$mice. As shown in Figure 1d, no differences were evident either in the kinetics or in the level of final mitochondrial polarization, when complexes I, III, IV or complexes II, III, IV were working. To test ATPase activity, we blocked the electron flow through the respiratory chain by inhibiting complex III with antimycin $A$, and observed the expected collapse of $\Delta \psi . \Delta \psi$ was then reestablished by adding ATP which triggered ATP hydrolysis and proton pumping by complex $\mathrm{V}$. Interestingly, under these circumstances, the mitochondria from $\mathrm{TG} 2^{-/-}$animals showed a significantly slower rate of membrane polarization
(Figure 1e). This defect reflected a failure in ATPase function. To confirm this, we directly measured complex V-ATP synthesis rates. As shown in Figure 1c, TG2 ${ }^{-1-}$ mice exhibited a defect in this reaction as well. These observations confirmed a mitochondrial functional defect in the $\mathrm{TG} 2^{-1-}$ mice, and suggested that alterations in the respiratory chains might have affected the global production of ATP in tissues.

These effects should be enhanced in those organs that are not predominantly glycolytic. Moreover, differences should be more pronounced after a prolonged physical challenge, when metabolic stores of energy are depleted and mitochondria are in charge for ATP production. To address this hypothesis, we measured the ATP content in the liver, in the skeletal and in 
the cardiac muscles. We did not detect any major difference under resting condition. On the contrary, a prolonged physical effort (mice were kept running for 10 min on Rota Rod at constant low speed and then the speed was progressively increasing until the mice fell down) resulted in a drastic depletion of ATP content in skeletal and cardiac muscle of TG2 knockout mice (data not shown). In particular, we observed a drastic decrease of about $40 \%$ of ATP levels in the heart of TG2 ${ }^{-1-}$ mice as compared to controls $(P<0.01)$. Noteworthy, $1 \mathrm{~min}$ after increasing the Rota Rod speed all the $\mathrm{TG}^{-1-}$ mice fell down, while the control animals were still well performing.

These data provide evidence for a novel function of TG2 participating in the maintenance of the intact mitochondrial respiratory function, the absence of which leads to a serious failure in ATP production. Defects observed in TG2 ${ }^{-1-}$ mice can be due both to a lack of direct action of TG2 on the mitochondrial substrate proteins as well as to the absence of TG2-catalyzed post-translational modification(s) of some important mitochondrial regulatory proteins. Recent evidence indicates that mitochondrial respiratory complexes are associated into functional super-complexes, thus defects in a given complex may affect the function of the others. Interestingly, the insulin secretion defect observed in these animals ${ }^{6}$ might, at least partially, be explained by the mitochondrial assembly defects described here, as this pathology has been also related to failure in ATP production ${ }^{8}$ In conclusion, we present compelling evidence that under physiological circumstances TG2 acts at mitochondrial level. Future work should determine the TG2 mitochondrial substrates and which of the many TG2 enzymatic activities (i.e. transamidating, kinase and protein disulphide activity) is responsible for the physiological function described in this study.

\section{Acknowledgements}

The authors thank Susanna Cotecchia for providing the $\mathrm{AR}^{-1-}$ mice. This work was partially supported by grants from: European Community (QLK-
CT-2002-01956 and QLK3-CT-2002-02017) 'APOCLEAR'; Ricerca corrente e finalizzata del 'Ministero della Salute'; progetto Genomica Funzionale COMETA; COFIN 2004 and FIRB-2001 from MIUR, Hungarian National Research Fund (OTKA T049445, TS44798, T'046145), Hungarian Ministry of Health (ETT 100/2003), RET-006/2004 and A.I.R.C.

Z Szondy ${ }^{\star, 1,7}, P G$ Mastroberardino 2,7 J Váradi ${ }^{3}$, MG Farrace ${ }^{2}, \mathrm{~N} \mathrm{Nagy}^{3}$, I Bak ${ }^{3}$, I Viti', MR Wieckowsk ${ }^{4,5}$, G Melino $^{6}$, R Rizzuto $^{4}$, Á Tósaki $^{3}$, L Fesus $^{1}$ and $M$ Piacentini

${ }^{1}$ Department of Biochemistry, Signaling and Apoptosis Research Group, Hungarian Academy of Sciences, Research Center of Molecular Medicine University of Debrecen, Debrecen, Hungary

2 Department of Biology, University of Rome 'Tor Vergata', Rome, Italy

3 Department of Pharmacology, University of Debrecen, Debrecen, Hungary

4 Department of Experimental and Diagnostic Medicine, University of Ferrara, Ferrara, Italy

${ }^{5}$ Nencki Institute of Experimental Biology, Warsaw, Poland

${ }^{6}$ Fondazione S. Lucia, Roma, Italy and Medical Research Council, Toxicology Unit, Hodgkin bld, Leicester, UK

7 SZ and PM have equally contributed to this paper.

* Corresponding author: Z Szondy, Department of Biochemistry, Signaling and Apoptosis Research Group, Hungarian Academy of Sciences, Research Center of Molecular Medicine University of Debrecen, Nagyerdei krt.98, Debrecen, Hungary. Tel: + 3652416432; Fax: + 3652314989;

E-mail: szondy@indi.dote.hu

1. Elsasser A et al. (2001) Basic. Res. Cardiol. 96: 219-226.

2. Okamura T et al. (2000) Cardiovasc. Res. 45: 642-650.

3. Fesus L and Piacentini M (2002) Trends Biochem. Sci. 27: 534-539.

4. Hasegawa $G$ et al. (2003) Biochem. J. 373: 793-803.

5. Mishra S and Murphy LJ (2004) J. Biol. Chem. 279: 23863-23868.

6. Bernassola $F$ et al. (2002) FASEB J. 16: 1371-1378.

7. Maechler $P$ and Wollheim CB (2001) Nature 414: 807-812.

8. Piacentini $\mathrm{M}$ et al. (2002) J. Neurochem. 81: 1061-1072.

9. Sarang Z et al. (2005) Hepatology 42: 578-587.

10. Beil FU et al. (1977) Eur. J. Biochem. 81: 151-164. 